\title{
Heart Rate Extraction from Novel Neck Photoplethysmography Signals
}

\author{
Irene García-López, Piyush Sharma and Esther Rodriguez-Villegas
}

\begin{abstract}
This paper demonstrates for the first time how heart rate (HR) can be extracted from novel neck photoplethysmography (PPG). A novel algorithm is presented, which when tested in neck PPG signals recorded from 9 subjects at different respiratory rates, obtained good precision with respect to gold standard ECG signals. Mean absolute error (MAE), standard deviation error (SDAE) and root-mean-square error (RMSE) resulted in 1.22, 1.54 and 1.98 beats per minute (BPM), respectively. $H R_{\text {neck }}$ estimation showed strong correlation $(\mathbf{R}=0.94)$ with reference $H R_{E C G}$. Good agreement between both techniques was also demonstrated by Bland-Altman analysis. The bias between mean HR paired differences was -0.16 BPM and 95\% limits of agreement (LoA) were $(-4.7,4.4)$. Comparatively, for widely used finger PPG, errors were slightly smaller (MAE=0.38 BPM, SDAE=0.48 BPM, RMSE=0.62BPM) and the correlation with reference ECG was also very close to $1(R=0.99)$. Bias of -0.04 BPM and $95 \%$ LoA $(-1.5,1.4)$, also showed high degree of agreement. However, these findings show the potential the neck could have as an alternative body location for wearable monitors, aiming to reduce the number of sensing sites whilst still providing access to a wide variety of physiological parameters.
\end{abstract}

\section{INTRODUCTION}

Photoplethysmography (PPG) is a non-invasive optical technique to estimate changes in blood volume, relying on the difference in light absorption between arterial and venous vasculature [1]. More precisely, oxyhemoglobin $\left(\mathrm{HbO}_{2}\right)$, present in oxygenated blood, absorbs more infrared (IR) light $(910 \mathrm{~nm})$, than red $(\mathrm{R})$ light $(660 \mathrm{~nm})$. The opposite applies for deoxyhemoglobin ( $\mathrm{Hb}$ ), in deoxygenated blood. Considering these absorption properties of the hemoglobin molecule, and shining the skin with R and IR LEDs, arterial blood pulsations can be captured by a photodetector. The resulting PPG signal is composed of two components, AC and DC. The AC reflects the periodic pumping activity of the heart; and the DC accounts for the absorption from other tissues, as well as from non-pulsatile arterial and venous blood [2].

PPG signals are commonly used in clinical and ambulatory monitoring, to measure peripheral blood oxygenation levels $\left(\mathrm{SpO}_{2} \%\right)$ by means of pulse oximeters. These have also become very popular for tracking heart rate (HR), mostly in consumer products, since PPG provides, an easy to attach, low cost, and cable free alternative to traditional electrocardiography (ECG) [3].

This work was supported in part by "La Caixa" Foundation (LCF/BQ/EU15/10350027) and in part by the European Research Council under Grant 724334.

I. García-López, P. Sharma and E. Rodriguez-Villegas are with the Wearable Technologies Lab, Department of Electrical and Electronic Engineering, Imperial College London, SW7 2BT, United Kingdom. E-mail: \{irene.garcia-lopez15,piyush.sharma14, e.rodriguez\}@imperial.ac.uk
Although the finger is considered the gold standard site for PPG, the alternative location of the neck, could be extremely advantageous to reduce the number of body sensing sites necessary when multiple physiological parameters are required, such as for example in the case of detection of apnea events with oxygen desaturations during sleep [4]. Indeed, the neck has a great potential to be the ideal site for multi-modal signal acquisition, from a single wearable system, when several cardio-respiratory parameters ought to be monitored simultaneously.

However, little attention has been paid to neck PPG in the literature. Previous works focused on the design of a proof of concept system to extract the PPG signal and $\mathrm{SpO}_{2} \%$ from this location [5], and on determining the most stable position for signal acquisition [6]. In our previous work [7], we characterized the neck PPG pulse waveform by means of different contour features and spectral content.

In this paper, we compare the accuracy of extracting HR from the neck PPG against reference ECG. HR estimation from finger PPG is evaluated similarly, in order to assess the potential of neck PPG with respect to the preferred location in pulse oximetry.

\section{METHODS}

\section{A. Data acquisition}

PPG, ECG and respiratory signals were acquired from a total of 9 healthy participants in supine position. The cohort was composed of 5 males and 4 females, with average age of $24 \pm 3$ years old, and BMI of $22.6 \pm 3.7 \mathrm{~kg} / \mathrm{m}^{2}$. The study was approved by the Local Ethics Committee of Imperial College London (ICREC ref.: 18IC4358), and written informed consent was obtained from all subjects.

Three different measurement systems were used for data acquisition. Neck PPG signals were acquired by a reflectance pulse oximeter sensor (8000R, Nonin) placed externally at the suprasternal space, and connected to a processing module (Xpod, Nonin). Standard finger PPG signals were obtained from a synchronized transmission pulse oximeter (Onyx II 9560, Nonin) with Bluetooth connectivity, placed on the left hand. Signals were sampled at a rate of $75 \mathrm{~Hz}$. Reference cardiac and respiratory signals were also obtained with a portable polysomnography (PSG) system (SOMNOscreen Plus, SOMNOmedics). This consisted of: 2-leads ECG, flow thermistor sensor, nasal pressure cannula, chest and abdomen impedance plethysmography (IP) bands, and a finger transmission PPG sensor placed on the right hand. The PSG system also allowed the possibility of adding markers, which were used for posterior synchronization with the Nonin PPG sensors. In order to verify that all signals were correctly 

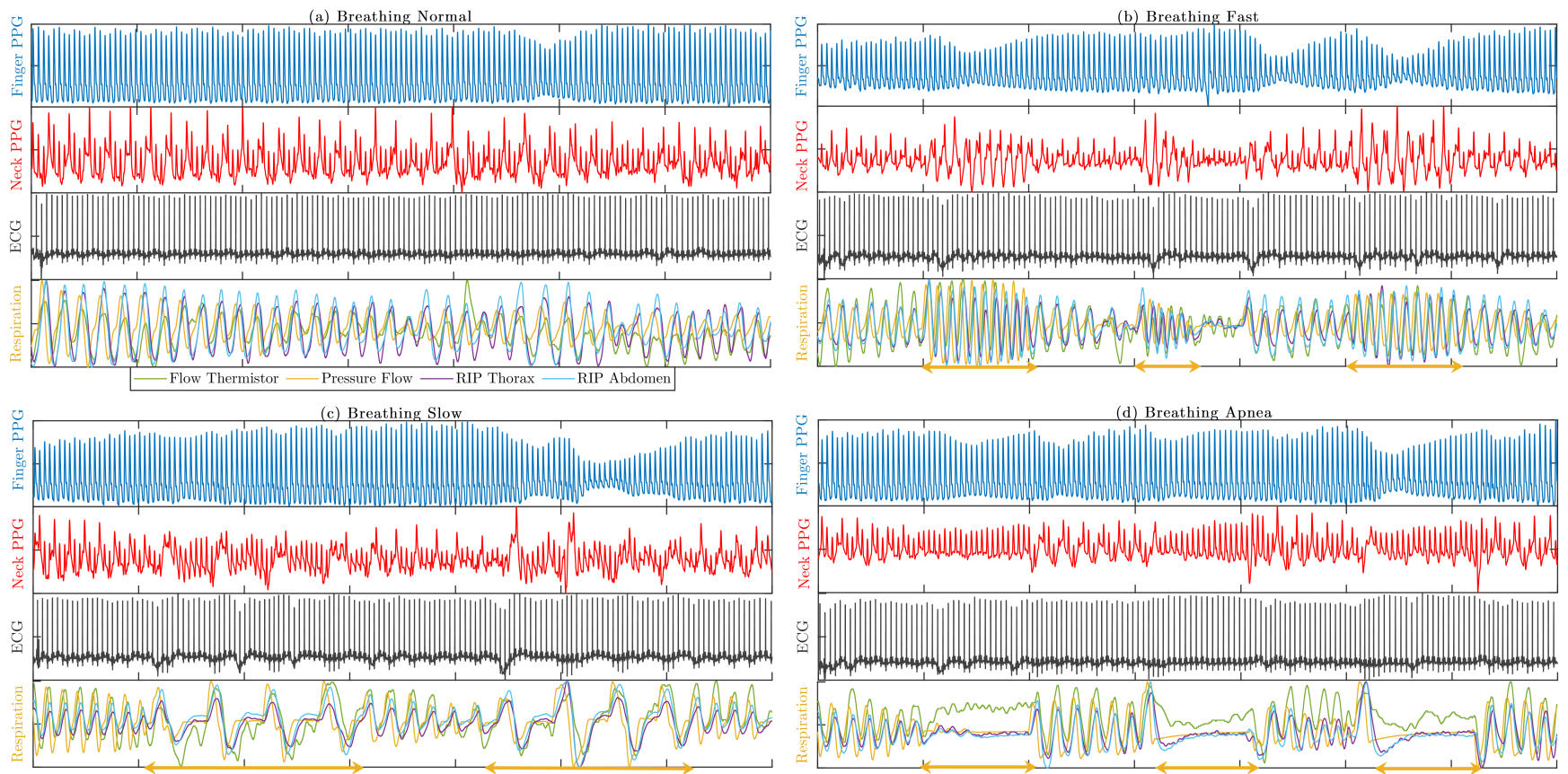

Fig. 1. (a) Normal, (b) Breathing Fast, (c) Breathing Slow, (d) Breathing Apnea, recordings for one subject showing all channels: finger PPG, neck PPG, ECG and respiratory sensors. Yellow horizontal arrows indicate the time interval of corresponding respiratory frequency modulation.

aligned in time for further processing, the correlation between finger PPG signals, from the Nonin and SOMNO systems, was obtained. If the initial synchronization did not maximize this correlation, a re-alignment was performed accordingly.

Initially in the experiments, participants were asked to breath at their normal pace for the first recording of 140s duration. The following three epochs were recorded also at rest but introducing different respiration frequency modulations of 20s duration: at fast pace, slow pace and simulated apnea events. These variations of respiratory rate were of interest since, according to the cardio-respiratory coupling, HR will vary accordingly. This hence allowed to test the response of neck PPG in a wider HR range, and therefore reproduce more realistic situations. All signals were normalized in the range of $[-1,1]$. Recordings of neck and finger PPG, ECG, and respiratory channels are shown, for different respiratory rates, in Figure 1. It is worth noting the effect of changes in breathing rate, pointed out by yellow horizontal arrows, on neck PPG signals.

\section{B. Heart beat detection and HR estimation}

HR was calculated for neck PPG, finger PPG and ECG channels in windows of $8 \mathrm{~s}$ with $6 \mathrm{~s}$ overlap. Since the total recording duration was of 140s, 67 windows were used. PPG signals were pre-processed with a 4th order high pass Butterworth filter with $0.7 \mathrm{~Hz}$ cut off frequency, in order to remove the DC and low frequency components. Systolic peaks of PPG pulses and R peaks of ECG signals were first detected in MATLAB 2018b using the findpeaks command. The minimum peak distance parameter was set to $0.6 \mathrm{~s}$, to ensure sufficient time resolution for large HRs (up to 100 BPM). A minimum peak amplitude threshold of -0.15 (a.u.) was also established. The cardiac frequency $H R_{w}$ estimate for each $8 \mathrm{~s}$ window $w=1,2, \ldots, 67$, was calculated in beats-per-minute (BPM), as:

$$
H R_{w}=\frac{60}{R R_{w}}
$$

where, $R R_{w}$ represents the average time interval between successive peaks in window $w$, and was computed as:

$R R_{w}=\frac{1}{N} \sum_{i=1}^{N} \Delta t_{\text {peaks }}(i)=\frac{1}{N} \sum_{i=1}^{N} t_{\text {peak }}(i+1)-t_{\text {peak }}(i)$

With $N$ representing the total number of inter-beat time differences $\Delta t_{\text {peaks }}$, in window $w$.

A correction step was included before definitive $H R_{w}$ storage, in order to amend spurious detected peaks that could negatively affect the precision of $R R_{w}$. For that, an adaptive average $\left(\mu_{\Delta \text { tpeaks }}\right)$ and standard deviation $\left(\sigma_{\Delta \text { tpeaks }}\right)$ of precedent inter-beat distances, were calculated and updated after each processed window. Every peak-to-peak time difference in the current window, was compared against an adaptive threshold such that:

$$
\left|\Delta t_{\text {peaks }}(i)-\mu_{\Delta t p e a k s}\right|>2.5 \sigma_{\Delta t_{\text {peaks }}}
$$

If the condition was true, the abnormal peak-to-peak difference $\Delta t_{\text {peaks }}(i)$ was discarded, and not taken into account for the updated calculation of $R R_{w}$, and ultimate $H R_{w}$ estimation. Figure 2 shows the neck PPG $\Delta t_{\text {peaks }}$ traces before (in gray) and after (in red) the correction for a normal breathing recording over time. The true ECG $\Delta t_{\text {peaks }}$ 


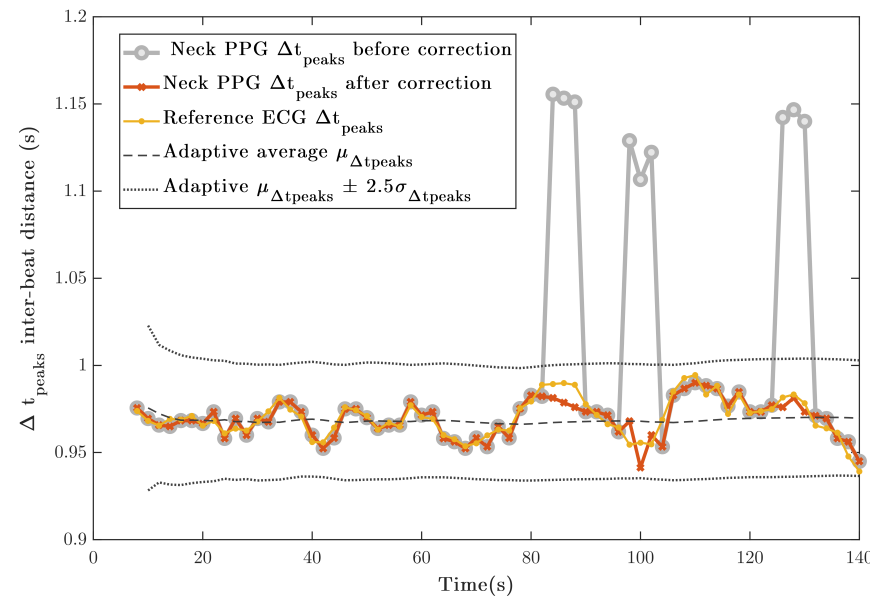

Fig. 2. Inter-beat distances over time of a breathing normal recording, before and after spurious peaks correction.

distances were also plotted for reference. It is noticeable how the correction reduced the distance between the neck PPG $\Delta t_{\text {peaks }}$ and the reference beat-to-beat ECG intervals.

\section{Performance metrics}

In order to evaluate the performance of HR extraction from PPG signals, several indices were used. Mean absolute error (MAE), standard deviation absolute error (SDAE) and root-mean-square error (RMSE) were calculated for each subject recording, and subsequently averaged out for the whole cohort. Additionally, $H R_{\text {neck }}$ or $H R_{\text {finger }}$ values were scattered against reference, $H R_{E C G}$, to find the best fitting using linear regression. Pearson's correlation coefficient (R) was also computed. Strongly positive (or negative) linear relationships would result in values close to 1 (or -1 ), whereas absence of correlation would output values in the proximity to 0 .

These metrics could only assess the distance and linear relationship between the two sets of PPG estimated and true ECG $H R_{w}$ observations. But, in order to adequately evaluate the degree of agreement between two quantitative methods measuring the same variable, a Bland-Altman analysis is commonly used [8]. For that, mean-differences of two measurement methods are plotted against reference method values to evaluate the bias. Limits of agreement (LoA) are also constructed to define the interval containing 95\% ( $\pm 1.96 \mathrm{SD})$ of the paired differences. In our case, $H R_{w}$ paired differences, $\left(H R_{n e c k}-H R_{e c g}\right)$ or $\left(H R_{\text {finger }}-H R_{e c g}\right)$, were graphically visualized against ECG true $H R_{E C G}$ values in Bland-Altman plots.

\section{RESULTS}

Cardiac frequency estimation from the novel PPG measurement site of the neck was tested and compared against the gold standard ECG method. For the sake of having a reference range of typical errors in conventional pulse oximetry, finger PPG signals were also used to extract HR and were compared to ECG values. A total of 31 recordings of 140s were evaluated, and 2077 HR estimates were extracted from each of the sensing modes. Results are presented in the following subsections.

\section{A. HR estimation errors}

Table I shows the HR performance between each PPG sensor modality and the ECG reference signals, in terms of errors and correlation coefficient. As it can be observed, HR estimation for the neck had in average a 1.22 BPM MAE, 1.54 BPM SDAE and 1.98 BPM RMSE. Pearson's correlation coefficient was very high $(\mathrm{R}=0.94)$ showing strong correlation between the estimated $H R_{\text {neck }}$ and true $H R_{E C G}$ values. For conventional finger PPG, MAE, SDAE and RMSE errors were even smaller with values of $0.38 \mathrm{BPM}$, 0.48 BPM and 0.62 BPM respectively. The correlation coefficient was also very close to $1(\mathrm{R}=0.99)$.

TABLE I

HR ESTIMATION PERFORMANCE COMPARISON FOR NECK AND FINGER PPG

\begin{tabular}{lcccc}
\hline PPG sensor & $\begin{array}{c}\text { MAE } \\
(\text { BPM })\end{array}$ & $\begin{array}{c}\text { SDAE } \\
(\text { BPM })\end{array}$ & $\begin{array}{c}\text { RMSE } \\
(\mathbf{B P M})\end{array}$ & R (correlation) \\
\hline Neck & 1.22 & 1.54 & 1.98 & $0.94(\mathrm{p}<0.01)$ \\
Finger & 0.38 & 0.48 & 0.62 & $0.99(\mathrm{p}<0.01)$ \\
\hline
\end{tabular}

\section{B. Linear regression fit}

HR PPG signals estimates from finger and neck sensors, were linearly fitted against true HR values of the reference ECG channel. The resulting linear models can be graphically observed in Figures 3(a-c), with their corresponding slopeintercept equations. In both cases, the predictor variables $\left(H R_{n e c k}\right.$ and $\left.H R_{\text {finger }}\right)$ suggest a strong positive linear relationship with the ground truth $H R_{e c g}$, as all data points seem to lie on the diagonal straight line with slope of 1 .

This is pretty much the case for the finger model, where the slope coefficient equals exactly 1 and the intercept has a very small value of $0.23 \mathrm{BPM}$. For the neck, the linear fit is still very close to ideal, but with a slightly higher intercept of 4.52 BPM, probably due to a larger spread of data. The coefficients of determination for neck and finger, $r^{2}=0.88$ and $r^{2}=0.99$ respectively, also suggest that the proportion of variance that each of the variables have in common with the ECG ground truth is very high.

\section{Bland-Altman analysis}

Bland-Altman graphical analysis for the paired differences of $H R_{n e c k}-H R_{E C G}$ and $H R_{\text {finger }}-H R_{E C G}$ are presented in Figures 3 (b-d). Novel neck PPG and reference ECG heart rates showed a good agreement with a bias of -0.16 BPM and $95 \%$ LoA of $(-4.7,4.4)$. The percentage of values found to lie beyond $\pm 1.96 \mathrm{SD}$ from the mean difference was 7\%. The conventional finger PPG and reference ECG cardiac frequencies demonstrated a bias of -0.04 BPM and $95 \%$ LoA $(-1.5,1.4)$, with $2.3 \%$ of paired difference scores beyond $\pm 1.96 \mathrm{SD}$. 

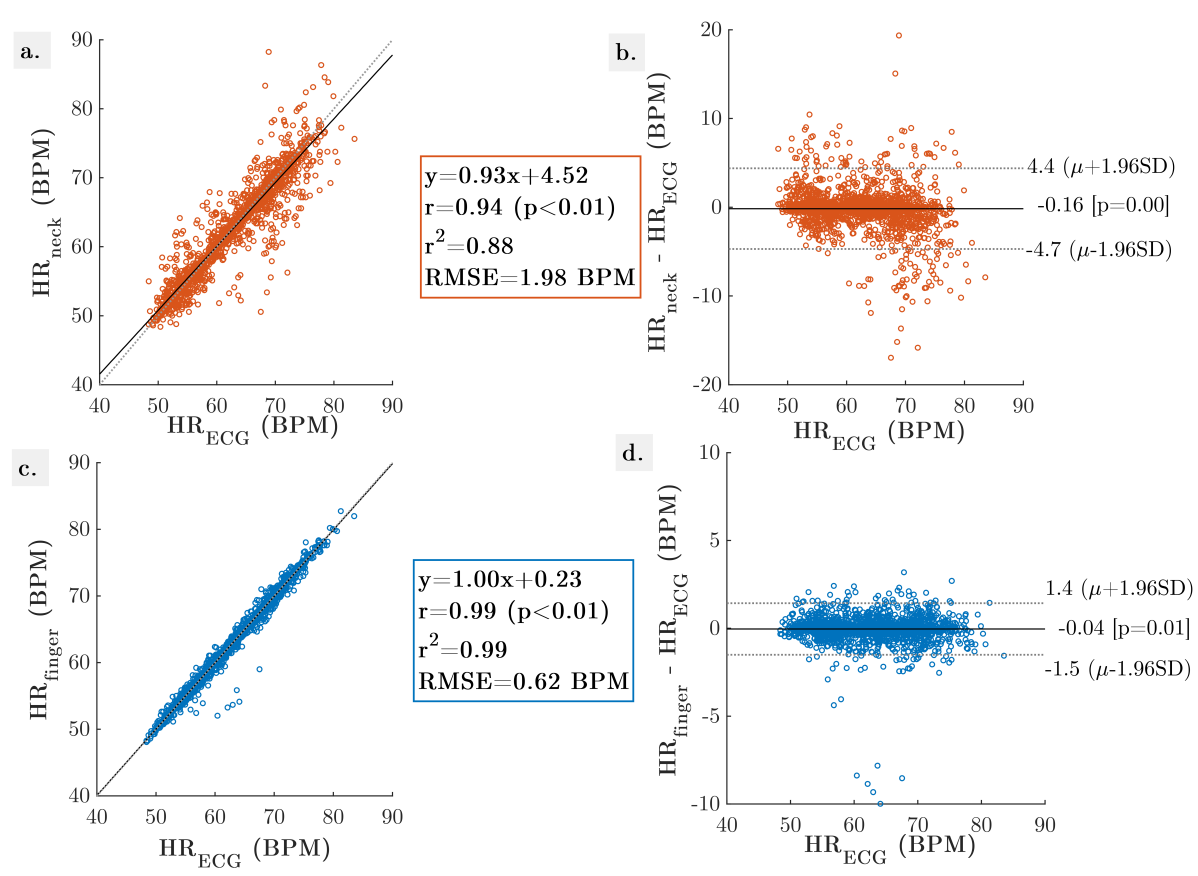

Fig. 3. (a-c). Linear regression of $H R_{n e c k}$ and $H R_{\text {finger }}$ estimation against $H R_{E C G}$. (b-d). Bland-Altman plots for all $H R$ estimates.

\section{DISCUSSION}

In this study, HR was extracted for the first time from novel neck PPG signals, at four different respiratory paces, in supine position. The developed algorithm precisely detected cardiac pulses relying on a mean inter-beat distances adaptive threshold for spurious peaks correction. HR was thus obtained in windows of $8 \mathrm{~s}$ with $6 \mathrm{~s}$ overlapping, by the inverse of peak-to-peak time differences multiplied by a factor of 60. The accuracy of $H R_{n e c k}$ extraction was evaluated with simultaneous ground truth ECG values. Average errors for all subjects and respiratory conditions, revealed very small differences between both techniques in the order of magnitude of $\sim 1$ BPM. This precision is very promising for accurate HR estimation. In addition, a linear regression model and Pearson's correlation coefficient demonstrated the strong linear relationship between neck PPG and ECG HR estimates. Ultimately, a Bland-Altman analysis showed a good agreement between both techniques, with very small bias between mean paired differences of HR observations, and $95 \%$ LoA of $(-4.7,4.4)$. These findings show the potential of neck PPG to reliably extract cardiac frequency with good accuracy.

When juxtaposing these results to the ones obtained for conventional finger PPG similarly, $H R_{\text {finger }}$ presented a reduced error and even higher similitude to ECG overall. Although this analysis was only included for comparative purposes within the same PPG modality, it is worth pointing out that, finger PPG is the widely established gold standard location in pulse oximetry to extract physiological parameters, whereas this work is still at the stage of proof of concept. Further work is required to test the proposed $H R_{n e c k}$ algorithm in different subject's sleep positions since this study was only carried out in controlled conditions as a first approach for the sake of simplicity. Similarly, artifacts that could severely disrupt the PPG signal quality should also be taken into account.

\section{CONCLUSIONS}

This work confirmed the suitability of the neck as an alternative body site for HR estimation based on PPG measurements. Future work should focus on estimating $H R_{\text {neck }}$ in the presence of artifacts to ensure its applicability in real life long term monitoring conditions. These advances could have a significant impact in the development of a unique wearable neck sensor incorporating multiple sensing modalities for multi-purpose cardio-respiratory applications.

\section{REFERENCES}

[1] J. Allen, "Photoplethysmography and its application in clinical physiological measurement," Physiological measurement, vol. 28, no. 3, p. R1, 2007.

[2] T. Tamura, Y. Maeda, M. Sekine, and M. Yoshida, "Wearable photoplethysmographic sensors: past and present," Electronics, vol. 3, no. 2, pp. 282-302, 2014.

[3] Y. Sun and N. Thakor, "Photoplethysmography revisited: from contact to noncontact, from point to imaging," IEEE Transactions on Biomedical Engineering, vol. 63, no. 3, pp. 463-477, 2016.

[4] E. Rodriguez-Villegas, G. Chen, J. Radcliffe, and J. Duncan, "A pilot study of a wearable apnoea detection device," BMJ open, vol. 4, no. 10, p. e005299, 2014.

[5] M. Peng, S. A. Imtiaz, and E. Rodriguez-Villegas, "Pulse oximetry in the neck-a proof of concept," in 39th Annual International Conference of the IEEE (EMBC). IEEE, 2017, pp. 877-880.

[6] Y. Zhong, Y. Pan, L. Zhang, and K.-T. Cheng, "A wearable signal acquisition system for physiological signs including throat ppg," in 38th Annual Conference of the IEEE (EMBC). IEEE, 2016, pp. 603-606.

[7] I. García-López, S. A. Imtiaz, and E. Rodriguez-Villegas, "Characterization study of neck photoplethysmography," in 40th Annual International Conference of the IEEE (EMBC). IEEE, 2018, pp. 4355-4358.

[8] D. G. Altman and J. M. Bland, "Measurement in medicine: the analysis of method comparison studies," The statistician, pp. 307-317, 1983. 